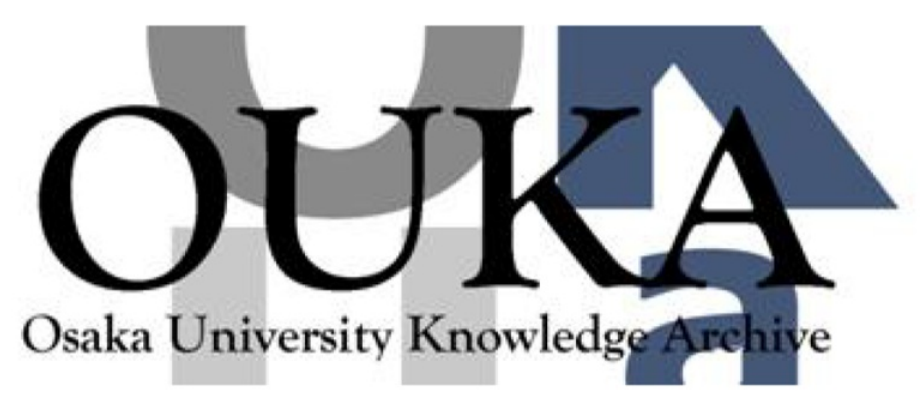

\begin{tabular}{|c|l|}
\hline Title & $\begin{array}{l}\text { Extraction of Product Evolution Tree from } \\
\text { Source Code of Product Variants }\end{array}$ \\
\hline Author(s) & Kanda, Tetsuya; Ishio, Takashi; Inoue, Katsuro \\
\hline Citation & \\
\hline Issue Date & $2013-08-26$ \\
\hline oaire:version & AM \\
\hline URL & $\begin{array}{l}\text { https://hdl. handle. net/11094/51561 } \\
\text { O 2013 ACM. This is the author's version of the } \\
\text { work. It is posted here for your personal use. } \\
\text { Not for redistribution. The definitive Version } \\
\text { of Record was published in SPLC'13 Proceedings } \\
\text { of the 17th International Software Product Line } \\
\text { Conference, Pages 141-150, 2013-08-26, } \\
\text { http://dx.doi.org/10.1145/2491627.2491637. }\end{array}$ \\
\hline rights \\
\hline Note & \begin{tabular}{l} 
\\
\hline
\end{tabular} \\
\hline
\end{tabular}

Osaka University Knowledge Archive : OUKA

https://ir. Library. osaka-u. ac. jp/

Osaka University 


\title{
Extraction of Product Evolution Tree from Source Code of Product Variants
}

\author{
Tetsuya Kanda, Takashi Ishio, Katsuro Inoue \\ Graduate School of Information Science and Technology, Osaka University \\ 1-5 Yamadaoka, Suita, Osaka, 565-0871, Japan \\ \{t-kanda, ishio, inoue\}@ist.osaka-u.ac.jp
}

\begin{abstract}
A large number of software products may be derived from an original single product. Although software product line engineering is advocated as an effective approach to maintaining such a family of products, re-engineering existing products requires developers to understand evolution history of the products. It is challenging because developers have only source code of products in typical cases. In this research, we propose to extract a Product Evolution Tree that approximates evolution history from source code of products. Our key idea is that two successive products are the most similar pair of products in evolution history. We construct a Product Evolution Tree as a minimum spanning tree whose cost function is defined by the number of similar files between products. As an experiment, we have extracted Product Evolution Trees from 6 datasets of open source projects. The result shows 53 to $92 \%$ of edges in the extracted trees are consistent with the actual evolution history of the projects.
\end{abstract}

\section{Categories and Subject Descriptors}

D.2.7 [Software Engineering]: Distribution, Maintenance, and Enhancement

\section{General Terms}

Experimentation

\section{Keywords}

software product line; software evolution; visualization

\section{INTRODUCTION}

Copying existing code fragments, source files, and the whole of a project is a common practice to develop a new software product [20]. For example, Linux kernel is forked into many projects including not only Linux distributions but also embedded software such as Android OS. Nonaka et al. analyzed corrective maintenance data of industrial embedded software products [17]. A part of the evolution history of the software product family is shown in Figure 1. The

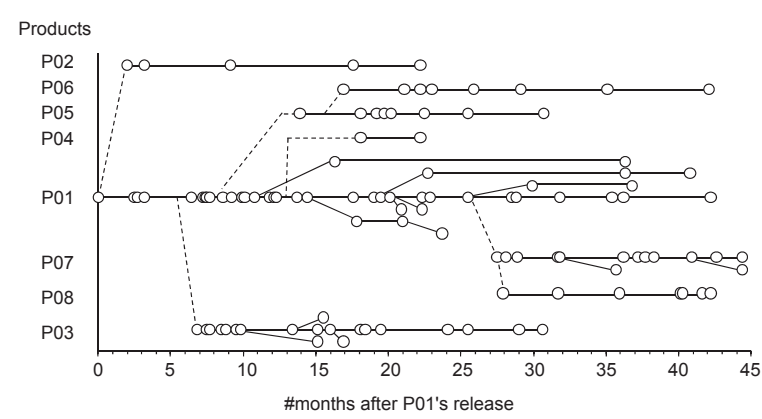

Figure 1: A product family derived from a single product $[17]$.

horizontal axis represents the number of months from the first release of the original product series (P01), the vertical axis represents product series ID in a company, respectively. In Figure 1, a circle corresponds to a product. Each dashed edge indicates that the new product series is derived from the original product. A solid edge connecting products indicates that the products are released as different versions of the same product series. Figure shows only 8 major product series and their variations, while the company had 25 series of products. Each series of products has 2 to 42 versions. Although Software Product Line Engineering (SPLE) is a famous approach to efficient maintenance of a software product family, industry already maintains a large number of derived software products without applying SPLE. Construction of a software product line from existing products is a very important problem and many re-engineering methods have been proposed $[2,11,26]$.

To construct a software product line, developers have to analyze and compare software products to identify commonality and variability in the products. Since analyzing a large number of software products is a hard task for developers, Krueger suggested that developers should start their analysis from a small number of software products, instead of all products at once [12]. Koschke et al. proposed an extension of reflexion method to construct a product line by incrementally analyzing products [11]. To follow these reasonable approaches, developers must choose representative software products as a starting point. If an evolution history of software products such as Figure 1 were available, developers could recognize the relationships among the products 
and choose representatives for their analysis. For example, developers could analyze the original product and the latest release on each branch. The selection would enable developers to identify core features and product specific features of the product family. However, such a history of products is often not available for developers [13]. In the worst case, developers have only source code of each product.

In this research, we propose to extract an approximated evolution history of software products from their source code. Our approach depends on only source code so that we can analyze products without version numbers, names or release dates. We define a Product Evolution Tree as a labeled tree whose each node represents a product, each edge connects similar products, and each label indicates similarity of products and direction of evolution, respectively. A Product Evolution Tree is computed as a minimum spanning tree. Its cost function is defined by the number of similar files between products. Similarity between files is computed by Yoshimura's function, which is based on the longest common subsequence of the files [25].

We have implemented our approach as a tool that takes as input source code and visualizes a Product Evolution Tree. Using the tool, we have conducted a case study with 6 datasets based on open-source projects. Whereas our approach is a simple algorithm, the result shows that 53 to $92 \%$ of edges ( $79 \%$ on average) are consistent with the actual evolution history.

Our contributions are summarized as follows:

- We have proposed a visualization technique of relationships among software products from their source code.

- Our tool and datasets are publicly available [27], so that other researchers can replicate and improve the approach.

In Section 2, we describe related work. Section 3 details our proposed approach. Section 4 shows the result of a case study. We discuss about the results in Section 5. Finally in Section 6, we describe conclusion and future work.

\section{RELATED WORK}

\subsection{Product Analysis}

To apply SPLE to existing similar software products, developers must analyze features in the products. Kastner et al. proposed CIDE to simplify software product line development [8]. CIDE requires a single software product and decomposes it into features. Duszynski et al. proposed a technique for analyzing multiple software system variants [1]. They extracted system structure model and variability model that represent commonality and variability of system variants from source code. Their technique allows for detailed goal-driven refinement of the analysis results.

To utilize these techniques for a large number of software products, developers must choose one or more software products for their analysis. Our approach enables developers to choose a starting point of their analysis by visualizing relationships among software products.

\subsection{Software Categorization}

Several tools have been proposed to automatically categorize a large number of software based on their domains such as compiler, database, editor, and so on. MUDABlue [9] classifies software based on similarity of identifiers in source code. MUDABlue employed latent semantic analysis which extracts the contextual-usage meaning of words by statistical computations. LACT [22] uses latent dirichlet allocation in which software can be viewed as a mixtures of topics. LACT used identifiers and code comments, but excluded literals and programming language keywords, to improve categorization. CLAN [16] focused on API calls. Its basic idea is that similar software uses the same set of APIs. While all of these tools are able to detect similar or related applications from a large software set, our approach focuses on very similar products derived from the same product, that are likely categorized into the same category by these tools.

\subsection{Software Evolution}

Yamamoto et al. proposed SMAT tool that calculates similarity of software systems by counting similar lines of source code [24]. They identify corresponding source files between two software systems using CCFinder [7], and then compute differences between file pairs. They applied their tool to a case study of software clustering, and extracted a dendrogram of BSD-UNIX Operation Systems. The dendrogram reported which OSs are similar to each other.

Tenev et al. introduced bioinformatics concepts into software variants analysis [21]. One of them is phylogenetic trees, which visualizes the similarity relations. They constructed dendrogram and cladogram from six BSD Unix family systems for example of phylogenetic trees.

Although their approaches and goals are similar to our idea, our approach visualizes more concrete relationships among products; which product was first released, which products were forked from the release, and so on.

\subsection{File-to-file similarity}

In many research, two or more software systems are compared with one another. When comparing software systems, similarity between source files is a very important metric. To find out the same or similar source code fragments, many code clone detection tools have been proposed [7, 14]. Using large-scale code clone detection techniques, Hemel and Koschke compared Linux kernel and its vendor variants [5]. They found vendor variants included various patches, but the patches are rarely submitted to the upstream. Another application of code clone detection is detecting file moves occurred between released versions of a software system [13].

Yoshimura et al. visualized cloned files in industrial products [25]. They have used an edit distance function as a source file similarity to find out cloned files whose contents are almost the same. We have employed their similarity function with an optimization and aggregated file similarity to product similarity.

Inoue et al. [6] proposed a tool named Ichi Tracker to investigate a history of a code fragment with source code search engines. It takes a code fragment as an input and extracts related code from source code search engines. It visualizes 
how related files are similar to the original code fragment and when they are released. Using the visualization, developers can identify the origin of the source code fragment or a more improved version of the code fragment. Our approach enables similar analysis on software products instead of source files.

We have assumed that two successive products are very similar to each other. This observation is shown by Godfrey et al.[3]. They detected merging and splitting of functions between two versions of a software system. Their analysis shows that a small number of software entities such as functions, classes or files are changed between two successive versions. Lucia et al. reported that most of bug fixes are implemented in a small number of lines of code [15]. Since these analysis reported that two successive versions are very similar, we infer that the most similar pairs of products are likely two successive versions. Although developers may modify a substantial number of lines of code to release a new version, the new version is likely more similar to the original version than future products derived from the new version.

\section{PRODUCT EVOLUTION TREE}

Product Evolution Tree is a tree that approximates evolution history. Each node of a tree represents a software product. Each edge indicates that a product is likely derived from another product. A label of an edge explains the cost of software changes between products and the direction of derivation: which product is an ancestor and which product is a successor. We construct a Product Evolution Tree from source code of products through 3 steps as follows.

1. We calculate file-to-file similarity for all pairs of source files of all products.

2. We construct a minimum-spanning tree of products. The cost between two products is based on the number of similar files between the products.

3. We put labels on edges based on the number of modified tokens between two products.

\subsection{File Similarity Calculation}

To calculate file similarity, we first normalize each of source files into a sequence of tokens. In a normalized file, each line has only a single token. We remove blanks from source files to avoid an impact of coding style. We also remove comments since they do not affect behavior of products. All other tokens including keywords, macros and identifiers are kept as is.

We calculate similarity for all pairs of files across different products, since a file may be renamed in a different product variant. To calculate similarity among source files, we follow Yoshimura's inter-file similarity analysis [25]. Given a pair of files $(a, b)$, their file similarity $\operatorname{sim}(a, b)$ is calculated using the normalized sequences $a_{t}$ and $b_{t}$ of the files as follows:

$$
\operatorname{sim}(a, b)=\frac{\operatorname{LCS}\left(a_{t}, b_{t}\right)}{\operatorname{LCS}\left(a_{t}, b_{t}\right)+A D D\left(a_{t}, b_{t}\right)+D E L\left(a_{t}, b_{t}\right)}
$$

where $\operatorname{LCS}\left(a_{t}, b_{t}\right)$ is the number of tokens in the longest common subsequence (LCS) between $a_{t}$ and $b_{t}$. A pair of $A D D\left(a_{t}, b_{t}\right)$ and $D E L\left(a_{t}, b_{t}\right)$ represents an edit distance from $a_{t}$ to $b_{t} ; D E L\left(a_{t}, b_{t}\right)$ is the number of deleted tokens which are unique to $a_{t}, A D D\left(a_{t}, b_{t}\right)$ is the number of added tokens which are unique to $b_{t}$, respectively. $A D D$ and $D E L$ can be represented as follows.

$$
\begin{aligned}
& A D D\left(a_{t}, b_{t}\right)=\operatorname{LENGTH}\left(b_{t}\right)-\operatorname{LCS}\left(a_{t}, b_{t}\right) \\
& \operatorname{DEL}\left(a_{t}, b_{t}\right)=\operatorname{LENGTH}\left(a_{t}\right)-\operatorname{LCS}\left(a_{t}, b_{t}\right)
\end{aligned}
$$

where $\operatorname{LENGTH(s)}$ is the number of tokens in $s$.

We have used a file similarity based on LCS, since we could optimize the calculation as described in Section 3.4. The following computation steps did not depend on the definition of file similarity function; hence, other methods such as code clone detection are also applicable to compute file similarity.

\subsection{Construction of Minimum Spanning Tree}

In this step, we construct a minimum spanning tree of products. To define a cost function for products, we count the number of similar file pairs. When the file pair has a higher similarity than a threshold, it is a similar file pair. We have used similarity threshold $t h=0.9$ in this paper, which is experimentally determined. The number of all possible similar file pairs $E_{s}$ and cost $C$ between software products $P_{1}$ and $P_{2}$ are defined as:

$$
\begin{aligned}
E_{s}\left(P_{1}, P_{2}, t h\right) & =\left\{(a, b) \mid a \in P_{1}, b \in P_{2}, \operatorname{sim}(a, b) \geq t h\right\} \\
C\left(P_{1}, P_{2}, t h\right) & =-\left|E_{s}\left(P_{1}, P_{2}, t h\right)\right| .
\end{aligned}
$$

It should be noted that cost is a negative value since it should be smaller if products have more similar file pairs.

After calculating cost, the result is an undirected weighted graph $G=(V, E) . V$ denotes that software products and $E$ connects them with the cost function $C$. We construct a minimum spanning tree $S=\left(V, E^{\prime}\right)$ of the graph $G . E^{\prime} \subset E$ is a set of edges which have the smallest total cost:

$$
\sum_{\left(P_{i}, P_{j}\right) \in E^{\prime}} C\left(P_{i}, P_{j}, t h\right) .
$$

We use Prim's algorithm [18]. In Prim's algorithm, an initial vertex is picked up at first. The vertex is a tree comprising a single vertex. Next, the algorithm lists up all edges connecting a vertex in the tree to a vertex outside of the tree, picks up one of the edges with the lowest cost, and includes the edge and its connected vertex in the tree. The process is repeated until all vertices are included in the tree.

Prim's algorithm allows any vertex as a starting point. In addition, if two or more edges have the same lowest cost, one of them can be arbitrary selected. In our implementation, we select a vertex or an edge depending on the input order.

\subsection{Calculation of Evolution Direction}

After a minimum spanning tree is constructed, we put labels on the edges. We hypothesize that source code is likely added rather than deleted when software evolves to the next version. To compute the relationship, we first define two functions for two products $P_{1}$ and $P_{2}$ as follows:

$$
\begin{aligned}
& A D D_{A L L}\left(P_{1}, P_{2}\right)=\sum_{(a, b) \in E_{s}\left(P_{1}, P_{2}, t h\right)} \operatorname{ADD}(a, b) \\
& D E L_{A L L}\left(P_{1}, P_{2}\right)=\sum_{(a, b) \in E_{s}\left(P_{1}, P_{2}, t h\right)} \operatorname{DEL}(a, b)
\end{aligned}
$$


Both functions approximate the total number of modified tokens in similar files between the products. We determine a direction label for an edge between products using the following conditions:

$$
\begin{cases}P_{1} \rightarrow P_{2}, & A D D_{A L L}\left(P_{1}, P_{2}\right)>D E L_{A L L}\left(P_{1}, P_{2}\right) \\ P_{1}=P_{2}, & A D D_{A L L}\left(P_{1}, P_{2}\right)=D E L_{A L L}\left(P_{1}, P_{2}\right) \\ P_{1} \leftarrow P_{2}, & A D D_{A L L}\left(P_{1}, P_{2}\right)<D E L_{A L L}\left(P_{1}, P_{2}\right)\end{cases}
$$

It is easy to get the amount of the changed source code because we have calculated how many tokens are added or deleted from one source file to another in the process of file similarity calculation.

\subsection{Optimization of File Similarity}

A naive computation of $\operatorname{sim}$ for $N$ files requires to compute LCS $N(N-1) / 2$ times. To reduce the computation time, we introduced an optimization that calculates sim value only if it is necessary.

The LCS of two files comprises only tokens included in both files. To estimate the length of LCS between files, we introduce the term frequency $t f(f, t)$ which represents how many times term $t$ appears in file $f$. For example, suppose two files $\mathrm{S} 1(\mathrm{AAABB})$ and $\mathrm{S} 2(\mathrm{ABBBB})$, where $\mathrm{A}$ and $\mathrm{B}$ are terms in the files. The term frequencies are $\operatorname{tf}(A, S 1)=3$, $t f(B, S 1)=2, t f(A, S 2)=1$ and $t f(B, S 2)=4$. Since the LCS can include at most one A and two Bs shared by the sequences, the maximum length of the LCS is 3. Indeed, the actual LCS between $\mathrm{S} 1$ and $\mathrm{S} 2$ is $\mathrm{ABB}$ whose length is 3. Comparing $\mathrm{S} 1(\mathrm{AAABB})$ with $\mathrm{S} 3(\mathrm{BBBAB})$ for another example, this pair also share one $A$ and two Bs but there are no LCS whose length is 3 . AB, BA and $\mathrm{BB}$ are possible LCS for them.

With term frequency, we can get maximum similarity

$$
\operatorname{msim}(a, b)=\frac{\sum_{t \in T} \min (t f(a, t), t f(b, t))}{\sum_{t \in T} \max (t f(a, t), t f(b, t))}
$$

of each file pair $(a, b) . T$ represents the set of terms appeared in all source files. The value of $\operatorname{sim}(a, b)$ equals to $m \operatorname{sim}(a, b)$ if all the common tokens appear in the same order in two sequences. If the order of tokens in a sequence is different from another sequence, then $\operatorname{sim}(a, b)$ is smaller than $m \operatorname{sim}(a, b)$. A fomula $m \operatorname{sim}(a, b) \geq \operatorname{sim}(a, b)$ is always true, hence we need to compute $\operatorname{sim}(a, b)$ only if $m \operatorname{sim}(a, b) \geq t h$. Although we have to scan a file to construct a term frequency vector, the vector is used $N-1$ times. In addition, time complexity of msim is $\mathcal{O}(|T|)$. It is much smaller than calculation of the LCS.

\section{CASE STUDY}

We have implemented our approach as a tool and conducted a case study. The goal of the case study is to evaluate how accurately a Product Evolution Tree recovers actual evolution history.

\subsection{Datasets}

We have prepared 6 datasets using open source projects. Each dataset comprises a set of products whose evolution history is publicly available. Table 1 shows the lists of products in the datasets. Column "\#" indicates the ID of the

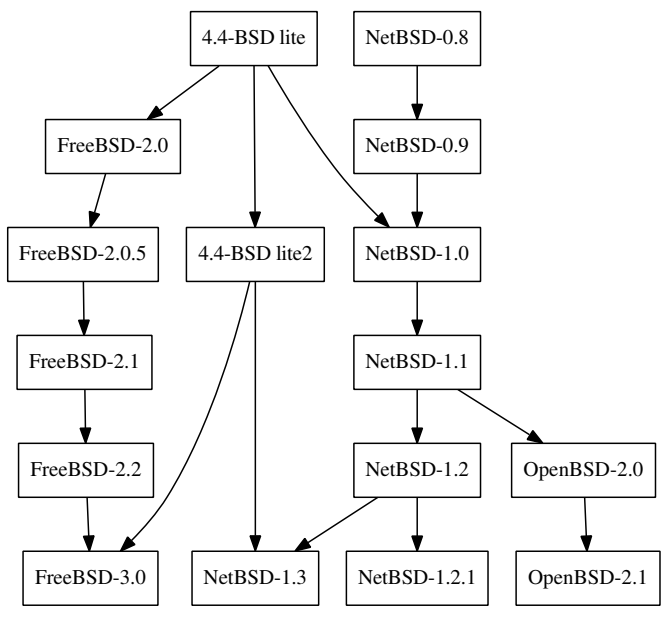

Figure 2: A family-tree of Dataset 6

dataset of each row. The other columns show the name of the dataset, products included in the dataset, the number of products, the total number of files and the total number of lines of code. Due to the limited space, we have omitted the intermediate version numbers in the table. For example, "8.0.0 - 8.0.26" indicates that the dataset included 27 version from 8.0.0 to 8.0.26. All datasets and the results are publicly available on our website [27].

In the datasets, we have used the following software.

PostgreSQL. It is a database management system. In the evolution history of PostgreSQL, each major version was released from the master branch after developing beta and RC releases. After a major version had been released, a STABLE branch was created for minor releases and the master branch was used for developing the next beta version. While each release archive contains a large amount of files, we used only source files under "src" directory in this case study.

FFmpeg and Libav. They are libraries and related programs for processing multimedia data. Libav is forked from FFmpeg and is developed by a group of FFmpeg developers. They are independently developed, but similar changes have been applied to both source code.

4.4BSD, FreeBSD, NetBSD and OpenBSD. These operating systems are derived from 4.3BSD, but they are now independent projects. The evolution history is publicly available as a "family-tree." Figure 2 shows a part of the family-tree for the versions selected for our dataset. According to the tree, NetBSD was originally derived from 4.3BSD, but NetBSD-1.0 is derived from 4.4BSD Lite. FreeBSD-2.0 is also based on 4.4BSD Lite. OpenBSD is forked from NetBSD. OpenBSD2.0 is its first official release. 4.4BSD Lite2 is the last release of $4.4 \mathrm{BSD}$ and it affects other BSD operating systems. In each version of distributed files, we used source files under "src/sys" directory. 
Table 1: Datasets

\begin{tabular}{|c|c|c|c|c|}
\hline \# Name & Included versions/tags & \#product & \#file & \#LOC \\
\hline 1 Pgsql-major & $\begin{array}{l}\text { PostgreSQL: } 7.0,7.1,7.2,7.3,7.4,8.0 .0,8.1 .0,8.2 .0,8.3 .0,8.4 .0, \\
9.0 .0,9.1 .0,9.2 .0\end{array}$ & $\overline{13}$ & 8,533 & $4,163,127$ \\
\hline $\begin{array}{ll}2 & \text { Pgsql8-all }\end{array}$ & $\begin{array}{l}\text { PostgreSQL: 8.0BETA } 1-8.0 \mathrm{BETA} 5,8.0 \mathrm{RC} 1-8.0 \mathrm{RC} 5 \\
\text { 8.0.0 - 8.0.26, 8.1BETA } 1-8.1 \mathrm{BETA} 4,8.1 \mathrm{RC} 1,8.1 .0-8.1 .23 \text {, } \\
\text { 8.2BETA } 1-8.2 \mathrm{BETA} 3,8.2 \mathrm{RC} 1,8.2 .0-8.0 .23, \\
\text { 8.3BETA } 1-8.3 \mathrm{BETA} 4,8.3 \mathrm{RC} 1-8.3 \mathrm{RC} 2,8.3 .0-8.3 .21, \\
\text { 8.4BETA } 1-8.4 \mathrm{BETA} 2,8.4 \mathrm{RC} 1-8.4 \mathrm{RC} 2,8.4 .0-8.4 .14, \\
\text { 8.5ALPHA } 1-8.5 \text { ALPHA } 3\end{array}$ & 144 & 96,448 & $48,478,395$ \\
\hline $\begin{array}{ll}3 & \text { Pgsql8-latest }\end{array}$ & $\begin{array}{l}\text { PostgreSQL: } 8.0 .20-8.0 .26,8.1 .17-8.1 .23,8.2 .17-8.2 .23 \text {, } \\
8.3 .15-8.3 .21,8.4 .8-8.4 .14,8.5 \text { ALPHA } 1-8.5 \text { ALPHA3 }\end{array}$ & 38 & 26,232 & $13,401,899$ \\
\hline 4 Pgsql8-annually & $\begin{array}{l}\text { PostgreSQL: } 8.0 .4,8.0 .9,8.0 .14,8.0 .18,8.0 .22,8.0 .26 \text {, } \\
8.1 .5,8.1 .10,8.1 .14,8.1 .18,8.1 .22,8.2 .5,8.2 .10,8.2 .14,8.2 .18,8.2 .22 \text {, } \\
8.3 .4,8.3 .8,8.3 .12,8.3 .16,8.3 .21,8.4 .1,8.4 .5,8.4 .9,8.4 .14\end{array}$ & 25 & 16,816 & $8,488,128$ \\
\hline $5 \quad$ FFmpeg & $\begin{array}{l}\text { FFmpeg(before fork): v0.5 - v0.5.3 } \\
\text { FFmpeg(after fork): n0.5.5 - n0.5.10 LibAV: v0.5.4 - v0.5.9 }\end{array}$ & 16 & 9,872 & $3,952,273$ \\
\hline 6 *-BSD & $\begin{array}{l}\text { BSD: 4.4BSD Lite, 4.4BSD Lite2 FreeBSD: } 2.0,2.0 .5,2.1,2.2,2.3 \\
\text { NetBSD: } 0.8,0.9,1.0,1.1,1.2,1.2 .1,1.3 \text { OpenBSD: } 2.0,2.1\end{array}$ & 16 & 6,204 & $6,050,462$ \\
\hline
\end{tabular}

Each dataset represents a particular situation of product analysis as follows.

Dataset 1: Pgsql-major. This is a dataset whose evolution history is straight, i.e., it has no project fork. The dataset contains 13 versions which are the first versions of each major release. We found that all of these releases are developed in the master branch. Hence, the resultant Product Evolution Tree should form a straight line.

Dataset 2: Pgsql8-all. This is a dataset whose evolution history is a tree of a single project. We created this dataset to emulate a practical case; a large number of products are derived from a single original product. This dataset contains 6 branches: five STABLE branches for 8.0.X to 8.4.X and the master branch developing three ALPHA releases for 8.5. It should be noted that these branches are developed in parallel; for example, some products are released from 8.0.X branch after 8.1.0. The dataset contains 144 versions in total.

Dataset 3: Pgsql8-latest. This is a dataset that includes only recent products. If a product family has a long history, older products may be obsolete to be included in a product line. In addition, such older products may be no longer available for developers. The dataset is a subset of Dataset 2 . It contains 38 versions including 7 latest versions in each of 5 STABLE branches and 3 releases in 8.5ALPHA series. This dataset contains no older releases indicating how STABLE branches have been created; therefore, it is difficult to extract the relationship among branches.

Dataset 4: Pgsql8-annually. This is another dataset that a full collection of products is not available. Dataset 4 contains 25 versions which have been released around September from 2005 to 2012. Extracting the relationship among branches is difficult for the dataset, since no major releases are contained in the dataset.

Dataset 5: FFmpeg. This is a dataset whose project has been forked to two projects. This dataset is created to evaluate whether our approach can recover the evolution history of forked projects or not. The dataset contains FFmpeg 0.5 series from 0.5 to 0.5 .3 before the fork and from 0.5.5 to 0.5.10 after the fork. 0.5.4 is not included since the tag has not been available in the repository. In addition, Libav 0.5.4 to 0.5 .9 are included in the dataset.

Dataset 6: BSD. This is a dataset whose project has been forked to more than three projects. This dataset is created to evaluate whether our approach can recover the complex evolution history of the projects or not. The evolution history of BSD operating systems is the most complex in our datasets. In addition, there are releases created by merging source code from more than one products that are derived from their single origin. Since our approach extracts only a tree, our approach must miss such edges.

\subsection{Results}

Our tool takes as input source code in a dataset and outputs a Product Evolution Tree. For each edge in a Product Evolution Tree, we have checked whether the edge connects versions as of the actual evolution history or not, and then checked the labeled direction for the matched edges.

The correctness of the edges and labels is shown in Table 2. Column "Matched Edges" shows how many edges are matched with the actual history without considering direction. In other words, we only checked the shape of the tree. Column "Matched Labels" shows how many correct edges have correct direction. Column "Recall" indecates proportion of correctly identified edges to edges in an actual evolution history. We did not calculate precision in this experiment, since precision and recall are always the same (Datasets 1-5) or higher value (Dataset 6). This is because the number of edges in a Product Evolution Tree is the same 
Table 2: Result

\begin{tabular}{l|r|r|rr|rr|r}
\hline Dataset & History & Output & \multicolumn{2}{|c|}{ Matched Edges } & Matched Labels & Recall \\
\hline $1^{\dagger}$ & 12 & 12 & 12 & $(100 \%)$ & 11 & $(91.7 \%)$ & $91.7 \%$ \\
\hline 2 (Fig. 4) & 143 & 143 & 136 & $(95.1 \%)$ & 128 & $(94.1 \%)$ & $89.5 \%$ \\
\hline $3^{\dagger}$ & 37 & 37 & 30 & $(81.1 \%)$ & 30 & $(100 \%)$ & $81.1 \%$ \\
\hline 4 (Fig. 5) & 24 & 24 & 20 & $(83.3 \%)$ & 20 & $(100 \%)$ & $83.3 \%$ \\
\hline 5 (Fig. 6) & 15 & 15 & 13 & $(86.7 \%)$ & 11 & $(84.6 \%)$ & $73.3 \%$ \\
\hline 6 (Fig. 7) & 17 & 15 & 12 & $(70.6 \%)$ & 9 & $(75.0 \%)$ & $52.9 \%$ \\
\hline
\end{tabular}

as or less than the number of edges in the actual evolution history.

The result shows that 53 to $92 \%$ of edges are consistent with the actual evolution history. This is very promising result. One important concern is what kind of errors are included in a Product Evolution Tree. Since developers do not know the actual evolution history, a wrong edge may lead developers to misunderstanding of the product family. To analyze errors, we have categorized incorrect edges in Product Evolution Trees into 5 patterns as follows. Patterns are shown in Figure 3. In Figure 3, each left graph shows an actual evolution history and each right graph shows an extracted Product Evolution Tree. Thin edges are the connections that exist in the actual history. Thick edges are the errors; solid edges connect exact products but indicate a wrong direction, dashed edges do not exist in the actual history, respectively.

P1: Version Skip. This pattern is found in three successive versions; two edges $v_{1}$ to $v_{3}$ and $v_{2}$ to $v_{3}$ are detected instead of a path from $v_{1}$ to $v_{3}$ via $v_{2}$. Figure 3(a) shows an example. This pattern happens when $v_{2}$ and $v_{3}$ have the same change cost from $v_{1}$ or the change cost between $v_{1}$ and $v_{3}$ are very small. For example, a small bug fix between $v_{2}$ and $v_{3}$ that modifies a few lines of code added for $v_{2}$ causes this pattern.

P2: Misalignment of Branch. An edge connects two branches but does not connect actually branched products. In Figure 3(b), there are two branches A and B. While version 1 of branch B (B1) was actually forked from version 1 of branch $A(A 1)$ in the evolution history, the origin of branch $\mathrm{B}$ was recognized as version 2 of branch A (A2). In this pattern, A2 is actually more similar to B1 than A1, since both A2 and B1 include the same changes from A1, such as bug fixes.

P3: Misdirection. An edge connects accurate products, but its label shows the reverse direction as shown in Figure 3(c). It happens when the size of source code or the number of source files decreased by several activities such as refactoring and deletion of dead code.

P4: Missing Merge. Our Product Evolution Tree cannot detect a merge of two products derived from a single product. In Figure 3(d), we can see that the Product Evolution Tree detects branching from version A1 to version $\mathrm{A} 2$ and $\mathrm{B} 1$ but cannot detect merging from version $\mathrm{B} 2$ to $\mathrm{A} 4$. When a software product forked into some software products, they are usually very similar.

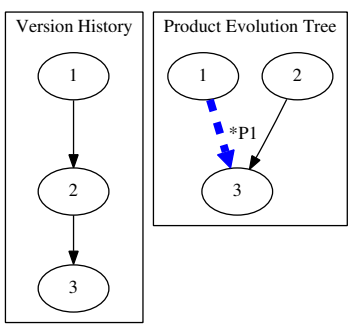

(a) P1: Version Skip

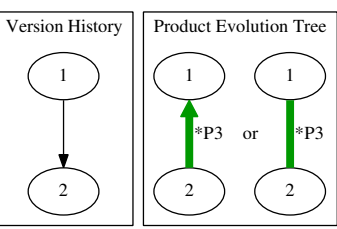

(c) P3: Misdirection

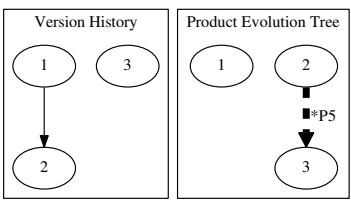

(e) P5: Out of Place

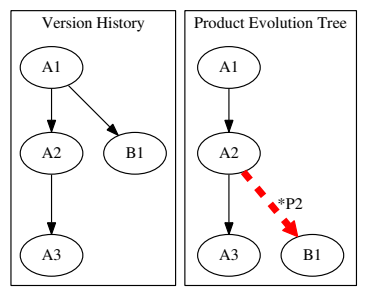
Branch

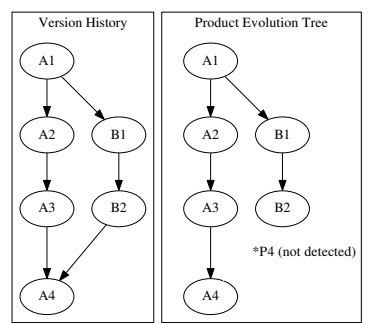

(d) P4: Missing Merge (b) P2: Misalignment of

\section{Figure 3: Patterns of incorrect edges}

Since the forked products are independently modified, their difference would be increased. Our approach connects similar products first, and a tree cannot have a closed path. Therefore, edges indicating project fork often appear in the tree but merges are hardly detected. In this pattern, one edge is missing but no wrong edges are output. If an actual evolution history includes a merge (e.g. Dataset 6 ), $100 \%$ recall is not achievable.

P5: Out of Place. This pattern is a falsely detected edge which is not classified into previous patterns. There are no relationship between the wrong edge and the actual history.

In these patterns, P1, P2 and P3 connected related products. P1 could be corrected by analyzing 3 related products. 
Table 3: The number of instances of incorrect edge patterns

\begin{tabular}{c|rrrrr|r}
\hline Dataset & P1 & P2 & P3 & P4 & P5 & Total \\
\hline 1 & & & 1 & & & 1 \\
\hline 2 & 1 & 4 & 8 & & 2 & 15 \\
\hline 3 & & 5 & & & 2 & 7 \\
\hline 4 & & 4 & & & & 4 \\
\hline 5 & 1 & 1 & 2 & & & 4 \\
\hline 6 & & 2 & 3 & 2 & 1 & 8 \\
\hline
\end{tabular}

P2 is not easily corrected, but it still connects two relevant branches. Therefore, developers might misunderstand only information that when the branching occurred. P3 could be corrected by manually comparing the edge direction with other edges around the products, and investigating difference between source code of the connected products. Therefore, these patterns are not so problematic errors. P4 is a missing edge; it is not correctable. Detecting merges is our future work. P5 is the most problematic error that connects irrelevant products.

Table 3 shows the number of pattern instances of incorrect edges. The table shows that a few problematic errors found in the datasets, although P2 and P3 are often included in the datasets.

\subsection{Product Evolution Trees}

In this section, we show the detail of each Product Evolution Tree extracted from the datasets. We compare a Product Evolution Tree with its actual history and then colored incorrect edges as indicated in Figure 3.

Dataset 1: Pgsql-major. The form of the tree is perfectly matched with the actual evolution history. But one label indicated the reverse direction on the edge 9.1.0-9.2.0. Among these releases, added source code is less than deleted source code, since several identifiers have been renamed and some refactoring has been performed. Even though the error exists, this Product Evolution Tree is still useful. For example, we can identify the latest version and the oldest version.

Dataset 2: Pgsql8-all. An overview of extracted Product Evolution Tree is shown in Figure 4. Since the tree is too large to show in the paper, a sequence of correctly connected versions is indicated by a single node. For example, the topleft node in Figure 4 represents 14 versions; 8.0.0BETA1 to 8.0.0BETA5, 8.0.0RC1 to 8.0.0RC5, and 8.0.0 to 8.0.3. In the figure, we annotated only incorrect edges and labels. The full version of this figure is also available on our website.

The Product Evolution Tree for Dataset 2 recovered its actual evolution history with high recall. However, almost all edges connecting branches are not matched. For example, 8.2BETA1 is developed on the master branch as the next version of 8.1.0. In the extracted tree, 8.2BETA1 is indicated as the next version of 8.1.5. We examined git repository and found that version 8.1 .5 is released right after 8.2BETA1. The master branch developing 8.2BETA1 and STABLE branch for 8.1 received the same 225 com-

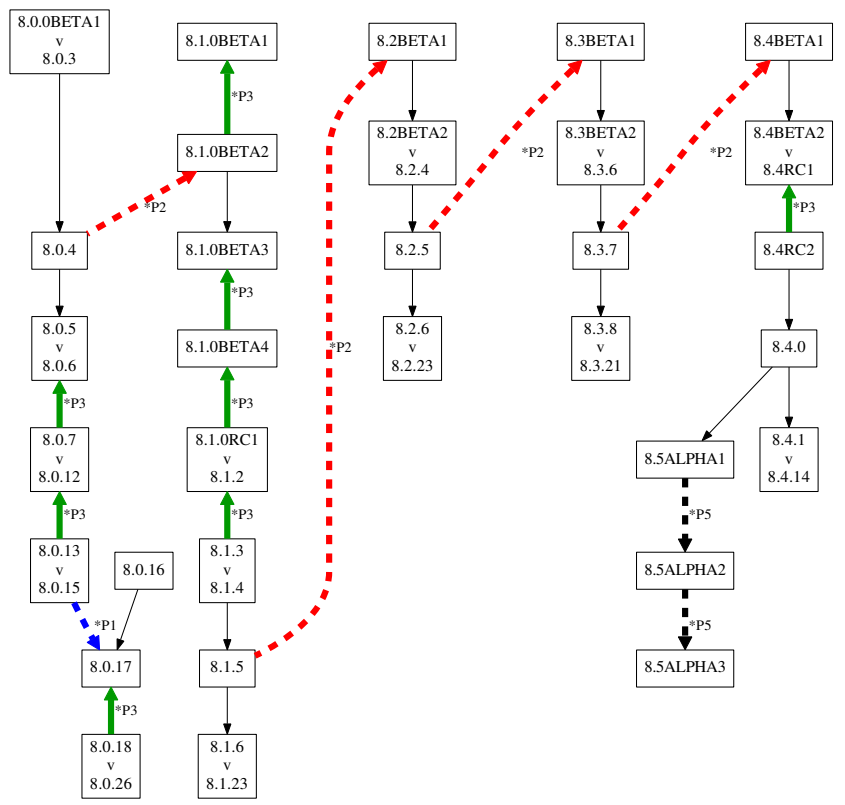

Figure 4: A Product Evolution Tree for Dataset 2

mits that are submitted in the same date with the same log message. During the same period (8.1.0 to 8.2BETA1), the differences between two branches are only 28 commits unique to the master branch. This fact means that the actual evolution history also does not always show functional differences of products.

Although the Product Evolution Tree is not perfect, we can find out 6 branches in the product set. For each branch, we can pick up the latest versions: $8.0 .26,8.1 .23,8.2 .23$, 8.3.21, 8.4.14 and 8.5ALPHA3. There are several labels in the branches indicated the reverse direction, but most edges indicated the correct evolution in the branches. Validating the direction of 143 edges of the tree requires much smaller effort than comparing arbitrary pairs of 144 products $(144 \times$ $143 / 2=10296$ pairs)

Dataset 3: Pgsql8-latest. The Product Evolution Tree of this dataset is almost the same as that of Dataset 2, except for edges connecting branches. Two STABLE branches often have the same changes, while only minor changes are unique to one of the branches. Therefore, intermediate versions of two branches are connected to each other (P2 instances). Nevertheless, the Product Evolution Tree shows the initial and latest versions of each branches, since there are a few error edges other than between branches.

Dataset 4: Pgsql8-annually. Figure 5 shows the Product Evolution Tree extracted from Dataset 4. Products are arranged horizontally if they are released on the same day. All edges and labels in the same branch are consistent with the actual evolution history. On the other hand, edges connecting between branches are mismatched. Since such inter-branch edges have larger cost value than edges inside a branch, we can identify the initial and latest versions of 


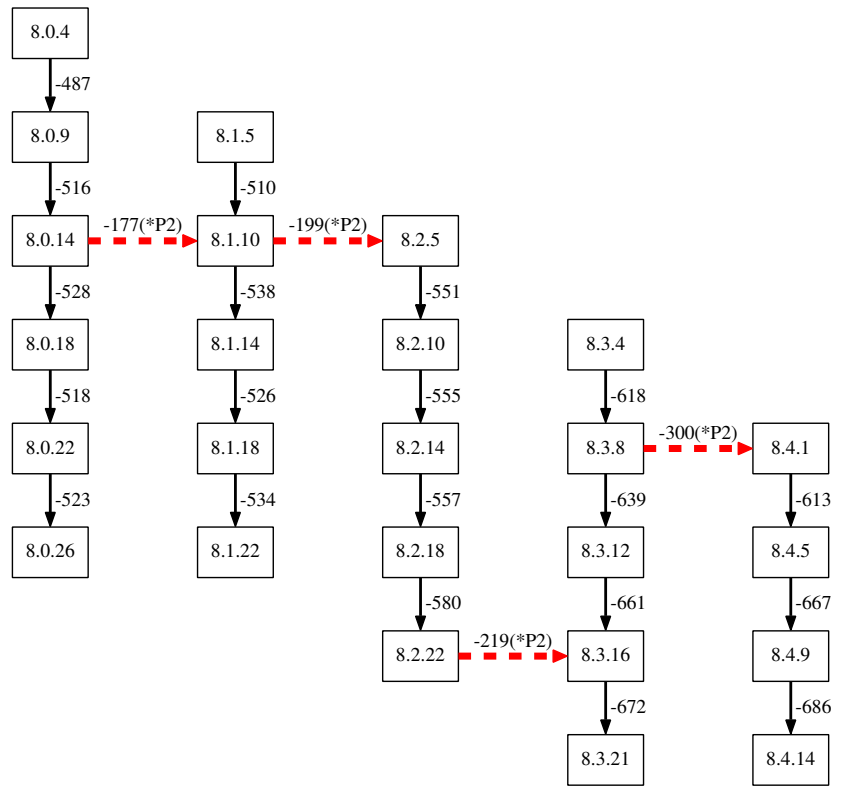

Figure 5: A Product Evolution Tree for Dataset 4

each branches in the Product Evolution Tree.

Dataset 5: FFmpeg. Figure 6 shows the Product Evolution Tree extracted from Dataset 5. The Product Evolution Tree does not correctly indicate when Libav was branched from FFmpeg project. FFmpeg 0.5.3 was branched into Libav 0.5.4 and FFmpeg 0.5.5 in the actual history, but the tree seems to indicate that FFmpeg 0.5.5 was derived from Libav 0.5.5. In those versions, projects shared the same changes, although they became independent projects.

Figure 6 also includes a wrong edge between FFmpeg 0.5 and 0.5.2. This edge is connected since files changed in 0.5.1 are changed again in 0.5 .2 , in other words, both versions have the same number of files changed from FFmpeg 0.5. This is the same phenomenon observed in Dataset 2.

The Product Evolution Tree shows Libav 0.5.5 is likely an origin of three branches. On that point of view, developers may choose the root version Libav 0.5.5 and leaf nodes FFmpeg 0.5, FFmpeg 0.5, FFmpeg 0.5.1, and Libav 0.5.9. The tree is still effective since the selected versions contain the actual original version and the latest versions. The tree reduces the effort for comparing all 15 versions.

Dataset 6: BSD. Figure 7 shows the Product Evolution Tree extracted from the dataset. Recall of this dataset was the worst in the all datasets, since the dataset included 17 correct edges but a Product Evolution Tree could include at most 15 edges.

The Product Evolution Tree included a merge relationship for NetBSD-1.0. It is the next release of NetBSD-0.9 including many source files from 4.4-BSD Lite. On the other hand, an edge from 4.4BSD Lite2 to FreeBSD-3.0 is not de-

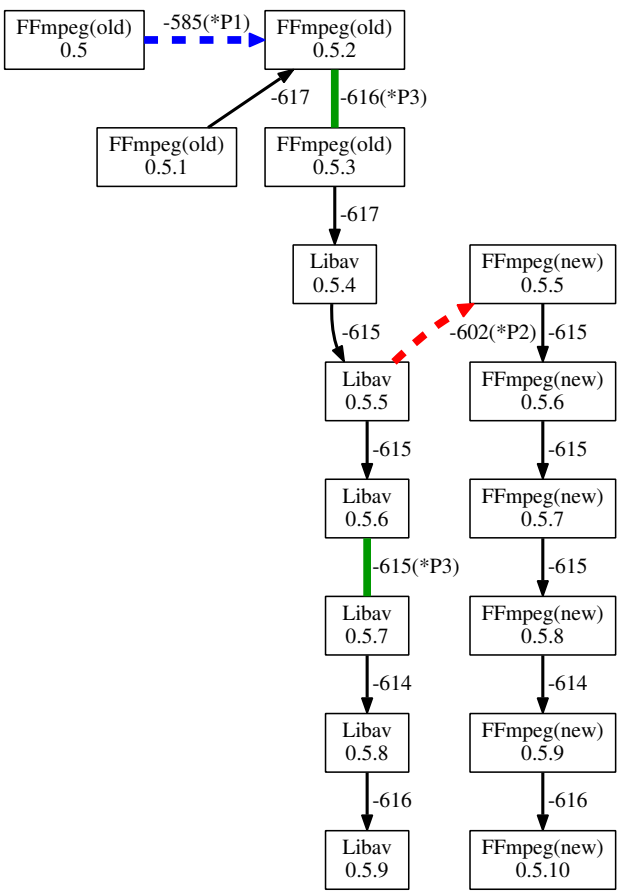

Figure 6: A Product Evolution Tree for Dataset 5

tected because Product Evolution Tree does not allow closed paths. In addition, the cost between two versions $C$ (4.4BSD Lite, FreeBSD-3.0, 0.9) $=-11$ indicated that all except for 11 files are different between two versions. The relationship from 4.4BSD Lite2 to FreeBSD-3.0 in the family tree may not be captured by the source code difference.

As an overall impression, NetBSD-1.0 and NetBSD-1.2 will get attention because they have three edges. Leaf nodes FreeBSD-3.0, NetBSD-0.8, NetBSD-1.3, and NetBSD-1.2.1 also seem important. The Product Evolution Tree suggests that 4.4-BSD Lite and OpenBSD-2.1 are not a characteristic release. It is hard to find out they are important releases in this dataset.

\section{DISCUSSION}

\subsection{Effectiveness of Product Evolution Tree}

The result shows that 70 to $100 \%$ of edges without labels and 53 to $92 \%$ of edges with labels are consistent with the actual evolution history. Almost all of the latest products of each branch are represented as leaf nodes, except OpenBSD-2.1 in Dataset 6.

One of major error patterns in Product Evolution Trees is $\mathrm{P} 2$. P2 is not a serious problem since edges connecting products between branches usually have larger cost than edges in a branch. Therefore, developers could easily recognize branches in a tree, even if their connections are not correct.

Another major error P3 is a counterexample for our hypothesis that "source code is likely added". We found two major reasons by analyzing the source code where a P3 appeared. The first reason is that refactoring such as class splitting and merging have been applied. Techniques for detecting refac- 


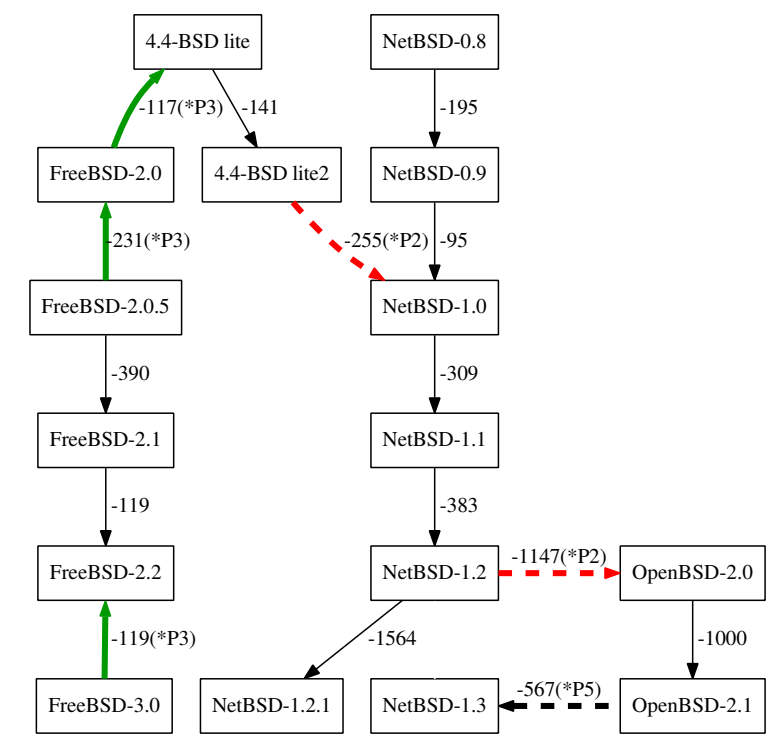

Figure 7: A Product Evolution Tree for Dataset 6

toring [23] may be helpful to remove incorrect labels caused by this reason. The second reason is non-essential changes [10]. Non-essential changes such as deleting dead code affect a large number of lines of code, while they are less important than other modification tasks such as feature enhancement. We can conjecture some cases that source code is decreased, but P3 was at most 20\% (3 of 15 in Dataset 6) of extracted edges in our case study. Hence, our method for determining the direction still worked effectively. It should be noted that we did not use information of the release dates since they are not always available. If release dates are available, all evolution direction would be correctly extracted if edges are correct.

Our approach always extracts $n-1$ edges for $n$ product variants as guaranteed by the algorithm. This is much smaller than $n(n-1) / 2$ comparison required if the relationships among the products have been completely lost. Even if the tree included wrong edges, most of the wrong edges connect somewhat related versions. Therefore, the tree would reduce the number of product pairs to be compared by developers.

A tree cannot have closed paths; as a consequence, we hardly detect merging of software like Dataset 6 . However, merging is not so frequent compared with forking, as shown in Figure 1. Since we could detect many forking in the case study, another analysis technique could be built to detect merging in the tree.

Although our approach can be applicable to arbitrary source code, intermediate versions are important clues to recover an evolution history. In Dataset 4, although several incorrect edges between branches (P2 instances) are included, other edges are completely detected. This is because minor releases in a single branch have small changes. In Dataset 1, a large number of lines of code is changed between two major releases; as a consequence, our approach extracted one wrong direction. A full collection of products may also be a cause of incorrect edges of P1 pattern, since two successive products are too similar to each other. We believe this is not a problem since developers can see similarity between products by a cost label.

From the shape of the Product Evolution Tree, developers can learn where is the starting point of the evolution and where they branched. Value of the cost function also provides hints to understand an evolution history. If the cost of an edge shows very small value than surrounding ones, there is only a slight difference and we can avoid comparing them. If a vertex has three edges and one of them has a higher cost than others, the high cost edge may indicate branching and others may indicate the mainline, respectively.

A Product Evolution Tree does not directly provide commonality and variability among products, but it will allow developers to use several analysis techniques. For example, Hashimoto et al. proposed to track change of source code in an evolution history [4]. They searched code clones in branches and mapping nodes of Abstract Syntax Tree among versions. Using the technique, the origin of source code can be estimated in the Product Evolution Tree. Rubin et al. proposed to search product features using the differences of source code [19]. Comparing the latest versions selected from a tree would be effective for developers to search features in a particular version.

We should discuss about scalability. To analyze Dataset 2, that is the largest dataset in this study, a machine equipped with two Intel Xeon E5507 processors (2.27 GHz, 4 cores) and 24GB RAM took about one day for analysis. We believe that it is reasonable cost for developers since product line analysis is unlikely an urgent task. In addition, file similarity and term frequency vectors can be computed in parallel. Furthermore, they are reusable for future analysis. For example, if new products are added, we can incrementally reconstruct a new Product Evolution Tree by comparing only new products and existing products.

\subsection{Threats to Validity}

Our assumption is that "two successive products are very similar to each other". On the other hand, developers may modify large amount of code and products might not be very similar to each other. The assumption is not always true but satisfied between many versions in our datasets.

Our algorithm for constructing the Product Evolution Tree is language-independent. However, all of the open source projects we have used in the case study are written in C. Source files in the datasets are well organized according to their functions. As a result, the number of edited files reflects the number of edited features; in other words, the number of similar files correctly reflects the distance between versions. If files are not clearly separated, our approach could be applied to such a program by dividing source code into functional unit such as subroutine or procedure.

Datasets 1, 2, 3 and 4 contain only PostgreSQL. Since there are some overlapped products, average accuracy of the results may be affected by source code of PostgreSQL than other projects. This threat is easily removed by additional experiments, since our tool needs only source code as input. 
We have used a single threshold 0.9 in the case study, which is determined by a small preliminary experiment. While it works for 6 datasets, a different threshold may be better for a different dataset.

\section{CONCLUSIONS}

Constructing SPLs from existing software products is becoming an important activity. In this paper, we proposed an automatic extraction of a Product Evolution Tree to help developers to understand evolution history of products. Our approach depends on only source code of products and connects similar software products based on the number of similar files. We have applied our tool to 6 datasets including several open source projects. As a result, 53 to $92 \%$ of edges are correctly recovered. The result is promising, since we can identify branches and the latest versions of products using a Product Evolution Tree, even if the tree included incorrect edges.

In future work, we must investigate whether the correctness is accurate enough for developers to analyze software products or not. In addition, we would like to try automated detection of software merge, so that we can extract a complete evolution history for arbitrary projects.

\section{ACKNOWLEDGMENTS}

This work is supported by KAKENHI (No.23680001).

\section{REFERENCES}

[1] S. Duszynski, J. Knodel, and M. Becker. Analyzing the source code of multiple software variants for reuse potential. In Proc. of WCRE, pages 303-307, 2011.

[2] D. Faust and C. Verhoef. Software product line migration and deployment. Software Practice and Experience, 33:933-955, 2003.

[3] M. Godfrey and L. Zou. Using origin analysis to detect merging and splitting of source code entities. IEEE Transactions on Software Engineering, 31(2):166-181, 2005.

[4] M. Hashimoto and A. Mori. A method for analyzing code homology in genealogy of evolving software. In Proc. of FASE, pages 91-106, 2010.

[5] A. Hemel and R. Koschke. Reverse engineering variability in source code using clone detection: A case study for linux variants of consumer electronic devices. In Proc. of WCRE, pages 357-366, 2012.

[6] K. Inoue, Y. Sasaki, P. Xia, and Y. Manabe. Where does this code come from and where does it go? integrated code history tracker for open source systems -. In Proc. of ICSE, pages 331-341, 2012.

[7] T. Kamiya, S. Kusumoto, and K. Inoue. CCFinder: a multilinguistic token-based code clone detection system for large scale source code. IEEE Transactions on Software Engineering, 28(7):654-670, 2002.

[8] C. Kästner, S. Apel, and M. Kuhlemann. Granularity in software product lines. In Proc. of ICSE, pages 311-320, 2008.

[9] S. Kawaguchi, P. K. Garg, M. Matsushita, and K. Inoue. MUDABlue: an automatic categorization system for open source repositories. Journal of Systems and Software, 79(7):939-953, 2006.
[10] D. Kawrykow and M. P. Robillard. Non-essential changes in version histories. In Proc. of ICSE, pages 351-360, 2011.

[11] R. Koschke, P. Frenzel, A. Breu, and K. Angstmann. Extending the reflexion method for consolidating software variants into product lines. Software Quality Journal, 17:331-366, 2009.

[12] C. W. Krueger. Easing the transition to software mass customization. In Proc. of PFE, pages 282-293, 2001.

[13] T. Lavoie, F. Khomh, E. Merlo, and Y. Zou. Inferring repository file structure modifications using nearest-neighbor clone detection. In Proc. of WCRE, pages 325-334, 2012.

[14] Z. Li, S. Lu, S. Myagmar, and Y. Zhou. CP-Miner: finding copy-paste and related bugs in large-scale software code. IEEE Transactions on Software Engineering, 32:176-192, 2006.

[15] Lucia, F. Thung, D. Lo, and L. Jiang. Are faults localizable? In Proc. of MSR, pages 74-77, 2012.

[16] C. McMillan, M. Grechanik, and D. Poshyvanyk. Detecting similar software applications. In Proc. of ICSE, pages 364-374, 2012.

[17] M. Nonaka, K. Sakuraba, and K. Funakoshi. A preliminary analysis on corrective maintenance for an embedded software product family. IPSJ SIG Technical Report, 2009-SE-166(13):1-8, 2009.

[18] C. R. Prim. Shortest connection networks and some generalizations. Bell System Technical Jounal, 36:1389-1401, 1957.

[19] J. Rubin and M. Chechik. Locating distinguishing features using diff sets. In Proc. of ASE, pages 242-245, 2012.

[20] J. Rubin, A. Kirshin, G. Botterweck, and M. Chechik. Managing forked product variants. In Proc. of SPLC, pages 156-160, 2012.

[21] V. Tenev and S. Duszynski. Applying bioinformatics in the analysis of software variants. In Proc. of ICPC, pages 259-260, 2012.

[22] K. Tian, M. Revelle, and D. Poshyvanyk. Using latent dirichlet allocation for automatic categorization of software. In Proc. of MSR, pages 163-166, 2009.

[23] P. Weißgerber and S. Diehl. Identifying refactorings from source-code changes. In Proc. of ASE, pages 231-240, 2006.

[24] T. Yamamoto, M. Matsushita, T. Kamiya, and K. Inoue. Measuring similarity of large software systems based on source code correspondence. In Proc. of PROFES, pages 530-544, 2005.

[25] K. Yoshimura and R. Mibe. Visualizing code clone outbreak: An industrial case study. In Proc. of IWSC, pages 96-97, 2012.

[26] K. Yoshimura, F. Narisawa, K. Hashimoto, and T. Kikuno. FAVE: factor analysis based approach for detecting product line variability from change history. In Proc. of $M S R$, pages 11-18, 2008.

[27] PRET-Extractor: http://sel.ist.osaka-u.ac.jp/pret/. 